\title{
Sintomas ansiosos e depressivos em universitários brasileiros
}

\author{
Juliana Maltoni ${ }^{1}{ }^{1}$ \\ Priscila de Camargo Palma ${ }^{1}$ \\ Carmen Beatriz Neufeld (1 1 \\ ${ }^{1}$ Faculdade de Filosofia, Ciências e Letras de Ribeirão Preto da Universidade de São Paulo, SP, Brasil.
}

\begin{abstract}
Resumo
Este estudo objetiva verificar a prevalência de sintomas ansiosos e depressivos em universitários e verificar se estas variáveis possuem associação com gênero, tipo de instituição, área e ano da graduação. Compõe a amostra 558 estudantes do interior de São Paulo (55,4\% mulheres) de faculdades pública e privadas, de diferentes áreas e períodos da graduação. Os instrumentos utilizados foram os Inventários de Ansiedade e de Depressão de Beck (BAI e BDI) e o Patient Health Questionnaire-9 (PHQ-9). Utilizou-se estatística descritiva e o teste Qui-Quadrado para análise dos dados. Os resultados demonstram que as faixas moderada e grave correspondem a $10,8 \%$ da amostra para o BAI, 5,4\% para o BDI e 5\% para o PHQ-9, com associação entre o gênero feminino e as faixas de gravidade de todos os instrumentos e o tipo de instituição e as faixas dos sintomas de ansiedade. O sofrimento psicológico da amostra e especificidades em relação ao gênero destacam a importância do aprimoramento do suporte oferecido pelas instituições universitárias.
\end{abstract}

Palavras-chave: estudantes universitários, ansiedade, depressão.

\section{Anxiety and depressive symptoms in brazilian college students}

\begin{abstract}
This study aims to characterize anxiety and depressive symptoms in college students and to verify if these variables are associated with sex, institution type, area and graduation year. The sample comprises 558 students from the interior of São Paulo state (55.4\% women), from public and private colleges, from different areas and graduation periods. Instruments used were the Beck Anxiety and Depression Inventory (BAI and BDI) and the Patient Health Questionnaire-9 (PHQ-9). Descriptive statistics and Chi-square test were used for data analysis. The results show that the moderate and severe ranges correspond to $10.8 \%$ of the BAI sample, $5.4 \%$ of the BDI and 5\% of the PHQ-9, with an association between sex and the scoring ranges for all instruments. Psychological suffering of the sample and gender specificities highlights the importance of improving the support offered by university institutions.
\end{abstract}

Keywords: college students, anxiety, depression.

\section{Sintomas ansiosos y depresivos en universitarios brasileños}

\section{Resumen}

Este estudio objetiva caracterizar los síntomas ansiosos y depresivos en universitarios y compruebar se estas variables se asociam con género, tipo de institución, área y año de graduación. Hace parte de la muestra 558 estudiantes del estado de San Pablo (55,4\% mujeres), de las facultades públicas y privadas, de las diferentes áreas y los períodos de graduación. Los instrumientos utilizados fueron los Inventarios de Ansiedad y Depresión de Beck (BAI y BDI) e Cuestionario de Salud del Paciente-9 (PHQ-9). Fuera utilizado de la estadística descriptiva y del prueba Qui-Quadrado para la evaluación de los datos. Los resultados demuestraran que las formas moderadas y graves corresponden 10,8\% de la muestra para el BAI, 5,4\% para el BDI y el 5\% para el PHQ-9, con la asociación entre el géneroy las formas de gravedad de todos los instrumientos. El sofrimiento psicológico de la muestra y las especificaciones en relación con el género destacan la importância del aumento del apoyo de las instituciones universitarias.

Palabras clave: estudiantes universitarios, ansiedad, depresión. 
O período compreendido entre os 18 e 25 anos corresponde à idade adulta emergente (Avnet, 2000, 2005). O início da idade adulta é considerado uma década usada para exploração ocupacional e relacional (Sadock \& Sadock, 2007), e a transição para este período pode ser um momento crítico. Como desafios centrais dessa etapa, espera-se do indivíduo a conclusão e o progresso em sua educação, bem como o ingresso no mercado de trabalho, garantindo sua independência do núcleo familiar. Uma parcela significativa desses adultos emergentes é universitária no Brasil, e quase $30 \%$ da população do país entre 18 e 24 anos frequenta o Ensino Superior, o equivalente a 7,3 milhões de brasileiros matriculados em cursos de graduação de mais de 2 mil instituições de ensino, 5,3 milhões em privadas (Instituto Nacional de Estudos e Pesquisas Educacionais Anísio Teixeira [INEP], 2013).

Os acontecimentos no período de adultez emergente influenciarão, a curto e longo prazo, a sociedade como um todo, considerando que as pessoas dessa faixa etária se tornarão a força de trabalho e os pais da próxima geração (Institute of Medicine and National Research Council [IMNRC], 2015). Os jovens desta faixa etária, no entanto, apresentam maior prevalência de riscos de problemas de saúde em comparação a adolescentes e adultos jovens (26 a 34 anos), com taxas maiores de estresse psicológico grave, ideações, planos e tentativas suicidas em relação a esse último grupo (Neinstein, 2013). Transtornos de ansiedade e depressão também são mais identificados nessa fase (Eisenberg, Gollust, Golberstein, \& Hefner, 2007), podendo ser mais frequentes do que na população geral, com taxas alarmantes demonstradas na literatura (Bayram \& Bilgel, 2008).

A American Psychiatric Association (APA, 2014) descreve para a população geral 2,9 e $7 \%$ de taxa de prevalência para ansiedade generalizada e transtorno depressivo maior, respectivamente. Em graduandos, a prevalência estimada acessada pelo Patient Health Questionnaire (PHQ) é de 4,2\% para transtornos ansiosos e 13,8\% para transtornos depressivos, 2,5\% reportando ideação suicida (Eisenberg et al., 2007). Quase o dobro de transtornos depressivos foi observado em universitários chilenos, em que $27 \%$ dos 800 entrevistados preencheram os critérios através do PHQ-9 (Baader et al., 2014). Ibrahim, Kelly, Adams e Glazebrook (2013) encontraram ainda, em sua revisão sobre o tema, uma taxa ponderada de $47,7 \%$ em estudos com mesmo instrumento. Os estudos da área utilizamse de diferentes instrumentos e metodologias, portanto, análises e comparações entre os resultados devem ser feitas com ponderação.
O Beck Depression Inventory (BDI) é indicado como o instrumento mais utilizado, com taxa ponderada de $24 \%$ em revisão da área (Ibrahim et al., 2013). Em estudo com 17.348 estudantes de 23 países, 20\% dos universitários que responderam ao BDI de 13 itens apresentaram sintomas moderados a severos de depressão $(\geq 8)$ (Steptoe, Tsuda, Tanaka, $\&$ Wardle, 2007). Níveis maiores foram encontrados em mulheres e indivíduos mais novos. Whisman e Richardson (2015) concluem que $12 \%$ dos 15.233 estudantes acessados pelo BDI-II pontuaram em níveis moderado ou acima $(\geq 20)$, taxa três vezes maior do que a encontrada em universitários sérvios através do BDI-IA (n=1940) (Simić-Vukomanović et al., 2016). Em estudo brasileiro com 200 participantes, essa porcentagem foi de $8,5 \%$, subindo para $16,5 \%$ quando avaliados os sintomas ansiosos pelo BAI (Brandtner \& Bardagi, 2009). Com mesmo instrumento, Alvi, Assad, Ramzan e Khan (2010) observaram 20,1\% (n=279) e Simić-Vukomanović et al. (2016) 10,8\% de sintomas ansiosos nessa faixa de gravidade.

Em relação aos demais instrumentos, observa-se a utilização frequente do Depression Anxiety Stress Scales (DASS), cujos resultados apresentam-se mais similares. Bayram e Bilgel (2008) apontam para 27\% de sintomas depressivos, Mahmoud, Staten e Lennie (2012) 29\% e Lovell, Nash, Sharman e Lane (2014) para 21,8\%. Respectivamente, a avaliação dos sintomas ansiosos demonstrou taxas de 47, 27 e $28,5 \%$. Como é possível observar, as taxas podem variar consideravelmente entre os diferentes estudos. Apesar disso, é evidente que os resultados demonstram que existe sofrimento psicológico importante nesta população estudada, e as consequências deste quadro são vastas.

A ansiedade e a depressão foram fatores reportados como impactantes no desempenho acadêmico por $21,8 \%$ e $13,5 \%$ dos estudantes acessados pelo National College Health Assessment - American College Health Association II (ACHA-NCHA II, 2014), respectivamente, com $8,1 \%$ de ideações suicidas. $\mathrm{O}$ sucesso acadêmico e o bem-estar são influenciados pela saúde mental, bem como a consequente adaptação dos indivíduos nesse contexto e etapa da vida (Hunt \& Einsenber, 2010). No entanto, pesquisas nacionais demonstram dificuldade de adaptação na graduação em $43 \%$ dos estudantes de universidades federais e $47,7 \%$ de crise emocional vivida no último ano. A procura por atendimento psicológico foi reportada por $29 \%$ e o psiquiátrico, por $9 \%$, aumentos significativos em relação a 2004 (Fórum Nacional de Pró-Reitores de Assuntos Comunitários e Estudantis [FONAPRECE], 2011).

Assim como na população geral (APA, 2014; Huot et al., 2013; Keita, 2007; Molina et al., 2014; Munhoz, 
Santos, \& Matijasevich, 2013; Sadock \& Sadock, 2007; Somers, Goldner, Waraich, \& Hsu, 2006; Viana \& Andrade, 2012), entre universitários, o sexo feminino também apresenta maiores escores e associação com ansiedade (Bayram \& Bilgel, 2008; Brandtner \& Bardagi, 2009; Eisenberg et al., 2007; Mahmoud, Staten, \& Lennie, 2012; Simić-Vukomanović et al., 2016) e depressão (Baader et al., 2014; Brandtner \& Bardagi, 2009; Ibrahim et al., 2013; Steptoe et al., 2007; Weitzman, 2004; Whisman \& Richardson, 2015).

Em relação ao ano da graduação, não há consenso quanto ao período mais crítico, com alguns autores apontando para o período inicial da graduação (Alvi et al., 2010; Bayram \& Bilgel, 2008; Brandtner \& Bardagi, 2009) e outros para a metade e final do curso (Draken, Hafen, Rush, \& Reisbig, 2012; Simić-Vukomanović et al., 2016). A área do curso parece ser uma variável menos explorada, mas algumas associações vêm sendo observadas, no entanto, as diferenças metodológicas e culturais dificultam comparações neste âmbito (SimićVukomanović et al., 2016). Estudantes de Ciências Sociais e Políticas demonstraram escores maiores em relação a cursos de Ciências Básicas, Engenharia ou Medicina (Bayram \& Bilgel, 2008) e, no Brasil, a área de Humanas, em relação à Ciências Sociais Aplicadas, Agrárias e da Saúde demonstrou níveis maiores de depressão (Brandtner \& Bardagi, 2009). As autoras propõem que as interações interpessoais inerentes a essa área possam favorecer maior sensibilidade e vulnerabilidade às questões emocionais. O tipo de instituição - pública e privada - e sua relação com a saúde mental de seus estudantes não foi uma variável investigada em nenhum dos estudos acessados.

Na perspectiva da saúde universitária, o importante papel das instituições nas redes de apoio dos estudantes deve ser sempre levado em conta, com suporte ofertado principalmente no início do curso e com a identificação dos estudantes de risco, uma vez que a maneira mais eficaz de prevenir a progressão de transtornos psiquiátricos primários é a intervenção precoce nesses indivíduos (Viana \& Andrade, 2012). Nesse sentido, Bardagi e Hutz (2012) propõem que a preocupação com a transição para o ingresso no ensino superior deveria começar nas escolas e se estender para a universidade.

A literatura indica alta prevalência de sintomas depressivos e ansiosos no contexto universitário. Os prejuízos causados pelo sofrimento psicológico e transtornos relacionados não possuem apenas impacto no âmbito acadêmico, podendo trazer consequências a curto e longo prazos tanto para os indivíduos quanto para a saúde pública como um todo. Dessa maneira, a importância da investigação da saúde mental universitária se mostra evidente, tanto para melhor compreensão da realidade brasileira e de variáveis que possam estar relacionadas - uma vez que o foco dos estudos geralmente recai apenas sobre prevalência e a variável sexo - como para o aprimoramento de programas de acolhimento/atendimento psicológico das instituições.

Este estudo possui como objetivo a caracterização de sintomas ansiosos e depressivos em uma amostra universitária do interior de São Paulo, a partir da análise da frequência dos itens e das faixas de gravidade dos instrumentos BDI, BAI e PHQ-9. Pretende-se investigar também a relação destes sintomas com o gênero, tipo de instituição (pública ou privada), área do curso e ano da graduação.

\section{Método}

\section{Participantes}

A amostra final foi composta por 558 graduandos de ambos os sexos, em diferentes etapas e áreas dos cursos da graduação, de qualquer idade e provenientes de universidades pública e privadas. Foram excluídos 64 participantes da amostra inicial devido ao não preenchimento de itens dos instrumentos. Os dados deste estudo são provenientes de um banco de dados estruturado posteriormente como parte de um projeto maior. Uma vez que foram contatados no ambiente universitário, de acordo com a disponibilidade dos mesmos, constitui-se uma amostra de conveniência.

\section{Instrumentos}

Inventário de Depressão de Beck (Beck Depression Inventory-BDI). Escala de autorrelato de Beck, Rush, Shaw e Emery, de 1982, com validação no Brasil (Cunha, 2001). É composto por 21 grupos de sintomas com quatro alternativas possíveis, variando entre escores de 0 a 3 em ordem crescente de agravamento dos sintomas. Reflete a sintomatologia dos últimos sete dias do indivíduo. A aplicação do BDI é indicada para indivíduos entre os 17 e 80 anos de idade. O escore total, feito a partir da soma dos itens, pode indicar diferentes níveis de intensidade de depressão entre os níveis: mínimo (0-11 pontos), leve (12-19), moderado (20-30) e grave (36-63).

Inventário de Ansiedade de Beck (Beck Anxiety Inventory - BAI). Escala constituída por 21 itens referentes a sintomas de ansiedade experimentados nos últimos sete dias, com respostas variando de 0 a 3 , refletindo níveis de gravidade em ordem crescente. $\mathrm{O}$ instrumento criado por Beck, Epstein, Brown e Steer, em 1988, também é adaptado para o português (Cunha, 2001). Recomenda-se a utilização deste instrumento 
a partir dos 17 anos. $\mathrm{O}$ escore total consiste na soma dos itens, classificado em níveis de intensidade: mínimo (0-10), leve (11-19), moderado (20-30) e severo (31-63).

Questionário Sobre a Saúde do Paciente (Patient Health Questionnaire - PHQ-9). O PHQ é um questionário autoaplicável desenvolvido e validado em dois estudos de Spitzer, Kroenke e Williams (1999) e Spitzer, Williams, Hornyak e McMurray (2000), a partir do (PRIME-MD). O PHQ-9 é o módulo de depressão do PHQ, composto por 9 itens com foco nos critérios diagnósticos do DSM-IV, cuja validade e confiabilidade foram analisadas por Kroenke, Spitzer e Williams (2001), constituindo-se um instrumento diagnóstico e que acessa a severidade dos transtornos depressivos. As respostas do instrumento variam de 0 a 3 em relação ao número de dias que o sintoma tem ocorrido nas duas últimas semanas. A severidade dos sintomas varia de acordo com o total: 0-4 - nada/ mínimo; 5-9 - leve; 10-14 - moderado; 15-19 moderadamente severo; 20-27 - severo. O diagnóstico de transtorno depressivo maior é realizado quando cinco ou mais sintomas estão presentes em pelo menos mais da metade dos dias. Além disso, o critério também é preenchido se um dos sintomas for humor deprimido ou anedonia. $\mathrm{O}$ instrumento e seu manual possuem uso livre e podem ser encontrados online ${ }^{1}$.

\section{Procedimento de coleta}

A coleta dos dados foi realizada entre 2011 e 2012 em universidades pública e privadas em uma cidade do interior de São Paulo. Primeiramente convidou-se as instituições de ensino para participação na pesquisa. Após o aceite das mesmas, os graduandos foram convidados em sala de aula e, aqueles que consentiram a participação através do Termo de Consentimento Livre e Esclarecido, responderam os questionários no local, com supervisão do aplicador. Como parte do presente trabalho, os dados coletados foram armazenados item a item em um banco de dados e posteriormente analisados.

\section{Questões éticas}

Este projeto foi aprovado pelo Comitê de Ética em Pesquisa (CAEE no 2010/11732-4). Os estudantes com escores considerados clinicamente significativos foram posteriormente notificados via telefone e informados sobre a importância de realizarem acompanhamento com um profissional e sobre os serviços de atendimento psicológico gratuito disponíveis na cidade.

\section{Resultados}

\section{Procedimento de análise dos dados}

Avaliou-se a frequência de cada item e faixa de gravidade dos sintomas de depressão e ansiedade. Verificou-se também a associação dos itens e das faixas em função das variáveis: gênero, ano da graduação, área do curso e tipo de instituição. A análise foi feita por meio de estatística descritiva e, para verificar a existência de associações, utilizou-se o teste Qui-quadrado, realizado pelo software $\mathrm{SAS}^{\circledR} 9.2 \mathrm{com}$ a PROC FREQ, com nível de significância adotado de 5\%.

\section{Descrição da amostra}

A amostra final foi constituída por 558 universitários (55,38\% mulheres) com idade média de 21 anos $(\mathrm{DP}=3,2)$, variando entre 16 e 40 anos de idade. Constituíram a amostra estudantes da área de Ciências Sociais Aplicadas (48\%), Saúde (29,21\%), Humanas (16,31\%) e Ciências Biológicas (6,45\%). A maioria dos estudantes eram pertencentes à faculdade pública $(70,61 \%)$ e cursavam os três primeiros anos da graduação $(32,08 \%$, $30,82 \%$ e $32,62 \%$, respectivamente). Apenas oito estudantes eram do $5 \mathrm{O}$ e dois do $6^{\mathrm{O}}$ ano de graduação.

Os sintomas clinicamente significativos (faixas moderada e grave) de ansiedade foram observados em $10,8 \%$ dos universitários $(\mathrm{M}=9,55 ; \mathrm{D} P=8,24)$ e os depressivos em 5,4\%, de acordo com o BDI ( $M=7,37$; $\mathrm{D} P=6,49)$, e $5 \%$ de acordo com o PHQ-9 ( $\mathrm{M}=5,72$; $\mathrm{D} P=4,77)$. Em relação a este instrumento, $6,1 \%$ dos indivíduos preenchem os critérios para diagnóstico de depressão maior. A ideação suicida foi reportada no BDI por 4,66\% dos indivíduos e por 5,73\% no PHQ-9.

Quanto à frequência de respostas dos itens dos instrumentos aplicados, observou-se que para os sintomas de ansiedade, $78,85 \%$ relataram ter sentido nervosismo nas últimas duas semanas, sendo a sensação mais pontuada. Sensação de calor $(63,2 \%)$ e incapacidade de relaxar $(57,89 \%)$ foram os outros itens mais pontuados, seguidos de medo que aconteça o pior (56,63\%); palpitação ou aceleração do coração (44,44\%); indigestão ou desconforto no abdômen (41,58\%); medo de perder o controle $(34,59 \%)$; suor (não devido ao calor) $(33,7 \%)$; dormência ou formigamento $(31,54 \%)$; assustado $(29,57 \%)$; atordoado ou tonto $(27,96 \%)$; tremores nas mãos $(27,24 \%)$; tremores nas pernas (24,9\%); trêmulo (22,22\%); medo de morrer (18,46\%); rosto afogueado $(17,74 \%)$; sensação de sufocação (17,57\%); sem equilíbrio (17,03\%); dificuldade de respirar $(15,41 \%)$; aterrorizado $(12,19 \%)$; e sensação de desmaio $(10,75 \%)$.

Os sintomas depressivos mais pontuados no BDI foram autocrítica $(54,3 \%)$, culpa $(54,12 \%)$ e falta de

\footnotetext{
1 Ver: www.phqscreeners.com.
} 
energia (50,54\%), seguidos de irritabilidade (49,46\%); dificuldade em tomar decisões $(41,76 \%)$; alterações no padrão do sono (41,58\%); diminuição do prazer $(39,78 \%)$; diminuição do interesse nos outros $(32,8 \%)$; dificuldade em trabalhar $(29,03 \%)$; tristeza $(28,32 \%)$; baixa autoestima (27,24\%); preocupação com a saúde $(26,7 \%)$; mudanças na autoimagem $(23,12 \%)$; desesperança $(22,22 \%)$; sentimentos de punição $(21,86 \%)$; diminuição do apetite $(20,07 \%)$; chorar $(18,46 \%)$; sensação de fracasso $(15,77 \%)$; perda de peso $(11,47 \%)$; diminuição do interesse sexual $(9,86 \%)$ e ideação suicida $(4,66 \%)$.

Através do PHQ-9, o sentimento de cansaço ou pouca energia foi o item mais pontuado, por $71,86 \%$ da amostra, seguido de pouco interesse ou pouco prazer em fazer as coisas $(61,83 \%)$; se sentir "para baixo"/deprimido(a)/sem perspectiva $(54,30 \%)$; falta de apetite/comendo demais (53,58\%); dificuldade para pegar no sono/permanecer dormindo/dormir mais do que de costume (50,54\%); dificuldade para se concentrar nas coisas $(45,16 \%)$; se sentir mal consigo mesmo/ achar que é um fracasso/que decepcionou a família ou a si mesmo (40,86\%); lentidão para se movimentar/ falar a ponto das outras pessoas perceberem, ou o oposto: estar agitado(a)/irrequieto(a) a ponto de andar de um lado para o outro muito mais do que de costume $(22,22 \%)$; pensar em se ferir de alguma maneira ou, que seria melhor estar morto $(5,73 \%)$. $\mathrm{O}$ diagnóstico para depressão maior realizado através do instrumento sugere que $6,1 \%$ dos graduandos encontram-se com o transtorno instalado. No entanto, nenhuma entrevista foi realizada a fim de verificar outras características necessárias.

\section{Associações entre as variáveis}

Foi rodada uma análise estatística a partir do teste do Qui-quadrado entre todos os itens e entre as faixas de gravidade obtidas através dos escores gerais em relação às variáveis: gênero, tipo de instituição, área do curso e ano da graduação. A Tabela 1 demonstra as associações em relação às faixas do BAI e as variáveis investigadas. Foi observada associação significativa entre as faixas de sintomas do instrumento em relação ao gênero e ao tipo de instituição, mas não para a área do curso e o ano da graduação.

$\mathrm{Na}$ análise item a item, a associação entre o gênero feminino e sintomas de ansiedade foi significativa para nove dos 21 itens avaliados pelo BAI, sendo estes: incapacidade de relaxar $(p<0,01)$; atordoado ou tonto $(p=0,02)$; palpitação ou aceleração do coração $(p=0,02)$; nervoso $(p<0,01)$; sensação de sufocação $(p=0,03)$; dificuldade de respirar $(p<0,01)$; medo de morrer $(p=0,03)$; indigestão ou desconforto no abdômen $(p<0,01)$; e sensação de desmaio $(p=0,02)$. O tipo de instituição privada demonstrou associação com cinco itens do instrumento: tremores nas pernas $(p=0,02)$; medo que aconteça o pior $(p=0,02)$; atordoado ou tonto $(p<0,01)$; tremores nas mãos ( $p=0,03)$; e medo de morrer $(p=0,04)$.

Em relação à área do curso, seis itens de ansiedade demonstraram associação significativa: dormência ou formigamento $(p<0,01)$; sensação de calor $(p=0,04)$; incapacidade de relaxar $(p=0,03)$; medo que aconteça o pior $(p<0,01)$; nervoso $(p=0,02)$; indigestão ou desconforto no abdômen $(p<0,01)$; e quatro itens em relação ao ano da graduação: sensação de calor $(p=0,02)$; medo que aconteça o pior $(p=0,03)$; ator-

TABELA 1

Associação entre as faixas de gravidade do BAI e as variáveis investigadas

\begin{tabular}{|c|c|c|c|c|c|}
\hline Variáveis & Minimo $n(\%)$ & Leve $n(\%)$ & Moderado $n(\%)$ & Severo $n(\%)$ & $p$ \\
\hline Total & $357(63,98)$ & $141(25,27)$ & $46(8,24)$ & $14(2,51)$ & \\
\hline Feminino & $178(49,86)$ & $89(63,12)$ & $32(69,57)$ & $10(71,43)$ & $<0,01 *$ \\
\hline Masculino & $179(50,14)$ & $52(36,88)$ & $14(30,43)$ & $4(28,57)$ & \\
\hline \multicolumn{6}{|l|}{ Instituição } \\
\hline Pública & $267(74,79)$ & $87(61,7)$ & $30(65,22)$ & $10(71,43)$ & $0,03 *$ \\
\hline Privada & $90(25,21)$ & $54(38,3)$ & $16(34,78)$ & $4(28,57)$ & \\
\hline \multicolumn{6}{|l|}{ Áreas } \\
\hline Sociais Aplicadas & $164(45,94)$ & $77(54,61)$ & $22(47,83)$ & $5(35,71)$ & 0,08 \\
\hline Saúde & $119(33,33)$ & $27(19,15)$ & $14(30,43)$ & $3(21,43)$ & \\
\hline Humanas & $54(15,13)$ & $24(17,02)$ & $8(17,39)$ & $5(35,71)$ & \\
\hline Biológicas & $20(5,6)$ & $13(9,22)$ & $2(4,35)$ & $1(7,14)$ & \\
\hline \multicolumn{6}{|l|}{ Ano } \\
\hline 1 & $119(33,33)$ & $42(29,79)$ & $14(30,43)$ & $4(28,57)$ & 0,59 \\
\hline 2 & $113(31,65)$ & $44(31,21)$ & $13(28,26)$ & $2(14,29)$ & \\
\hline 3 & $104(29,13)$ & $53(37,59)$ & $18(39,13)$ & $7(50)$ & \\
\hline 4 & $12(3,36)$ & $1(0,71)$ & $1(2,17)$ & $1(7,14)$ & \\
\hline 5 & $7(1,96)$ & $1(0,71)$ & 0 & 0 & \\
\hline
\end{tabular}


doado ou tonto $(p=0,03)$; e suor não devido ao calor $(p=0,04)$. A Tabela 2 demonstra as associações em relação às faixas do BDI e as variáveis investigadas, demonstrando que houve associação significativa apenas em relação ao gênero.

Considerando a análise entre os itens, oito dos 21 do BDI foram significativos para a variável gênero feminino: autocrítica $(p=0,02)$; chorar $(p<0,01)$; irritabilidade $(p<0,01)$; mudanças na autoimagem $(p=0,03)$; alterações no padrão do sono $(p<0,01)$; cansaço/diminuição energia $(p<0,01)$; diminuição do apetite $(p<0,01)$ e do interesse sexual $(p<0,01)$. Apenas o item de desesperança avaliado pelo BDI demonstrou esta associação com o tipo de instituição pública $(p<0,01)$. Sete itens tiveram associação significativa com a área do curso: desesperança $(p<0,01)$; autocrítica $(p=0,03)$; dificuldade em tomar decisões $(p=0,04)$; em trabalhar $(p=0,04)$; alterações no padrão do sono $(p<0,01)$; diminuição do apetite $(p=0,01)$; e do interesse sexual $(p<0,01)$; e nenhum em relação ao ano da graduação. Para os resultados do PHQ-9, apenas a variável gênero demonstrou associação significativa com as faixas do instrumento, como demonstrado na Tabela 3.

TABELA 2

Associação entre as faixas de gravidade do BDI e as variáveis investigadas

\begin{tabular}{|c|c|c|c|c|c|}
\hline Variáveis (n) & Minima n(\%) & Leve $n(\%)$ & Moderado n(\%) & Grave n(\%) & $p$ \\
\hline Total & $435(77,96)$ & $93(16,67)$ & $29(5,2)$ & $1(0,18)$ & \\
\hline Feminino & $228(52,41)$ & $60(64,52)$ & $20(68,97)$ & 1 & $0,05^{*}$ \\
\hline Masculino & $207(47,59)$ & $33(35,48)$ & $9(31,03)$ & 0 & \\
\hline \multicolumn{6}{|l|}{ Instituição } \\
\hline Pública & $308(70,8)$ & $65(69,89)$ & $20(68,97)$ & $1(100)$ & 0,92 \\
\hline Privada & $127(29,2)$ & $28(30,11)$ & $9(31,03)$ & 0 & \\
\hline \multicolumn{6}{|l|}{ Áreas } \\
\hline Sociais Aplicadas & $210(48,28)$ & $45(48,39)$ & $13(44,83)$ & 0 & 0,08 \\
\hline Saúde & $136(31,26)$ & $22(23,66)$ & $5(17,24)$ & 0 & \\
\hline Humanas & $62(14,25)$ & $21(22,58)$ & $7(24,14)$ & $1(100)$ & \\
\hline Biológicas & $27(6,21)$ & $5(5,38)$ & $4(13,79)$ & 0 & \\
\hline \multicolumn{6}{|l|}{ Ano } \\
\hline 1 & $136(31,26)$ & $30(32,26)$ & $13(44,83)$ & 0 & 0,9 \\
\hline 2 & $141(32,41)$ & $26(27,96)$ & $5(17,24)$ & 0 & \\
\hline 3 & $138(31,72)$ & $32(34,41)$ & $11(37,93)$ & $1(100)$ & \\
\hline 4 & $12(2,76)$ & $3(3,23)$ & 0 & 0 & \\
\hline 5 & $6(1,38)$ & $2(2,15)$ & 0 & 0 & \\
\hline 6 & $2(0,46)$ & 0 & 0 & 0 & \\
\hline
\end{tabular}

TABELA 3

Associação entre as faixas de gravidade do PHQ-9 e as variáveis investigadas

\begin{tabular}{|c|c|c|c|c|c|}
\hline Variáveis (n) & Leve $n(\%)$ & Moderado $n(\%)$ & $\begin{array}{c}\text { Moderadamente } \\
\text { Severo } n(\%)\end{array}$ & Severo $n(\%)$ & $P$ \\
\hline Total & $177(31,72)$ & $72(12,9)$ & $17(3,05)$ & $11(1,97)$ & \\
\hline Feminino & $104(58,76)$ & $44(61,11)$ & $13(76,47)$ & $8(72,73)$ & $0,04 *$ \\
\hline Masculino & $73(41,24)$ & $28(38,39)$ & $4(23,53)$ & $3(27,27)$ & \\
\hline \multicolumn{6}{|l|}{ Instituição } \\
\hline Pública & $130(73,45)$ & $50(69,44)$ & $9(52,94)$ & $8(72,73)$ & 0,5 \\
\hline Privada & $47(26,55)$ & $22(30,56)$ & $8(47,06)$ & $3(2,27)$ & \\
\hline \multicolumn{6}{|l|}{ Áreas } \\
\hline Sociais Aplicadas & $81(45,76)$ & $34(47,22$ & $9(52,94)$ & $4(36,36)$ & 0,26 \\
\hline Saúde & $47(26,55)$ & $18(25)$ & $3(17,65)$ & $2(18,18)$ & \\
\hline Humanas & $34(19,21)$ & $14(19,44)$ & $4(23,53)$ & $4(36,36)$ & \\
\hline Biológicas & $15(8,47)$ & $6(8,33)$ & $1(5,88)$ & $1(9,09)$ & \\
\hline \multicolumn{6}{|l|}{ Ano } \\
\hline 1 & $59(33,33)$ & $23(31,94)$ & $4(23,53)$ & $5(45,45)$ & 0,96 \\
\hline 2 & $55(31,07)$ & $19(26,39)$ & $5(29,41)$ & $1(9,09)$ & \\
\hline 3 & $57(32,2)$ & $26(36,11)$ & $6(35,29)$ & $5(45,45)$ & \\
\hline 4 & $4(2,26)$ & $3(4,17)$ & $1(5,88)$ & 0 & \\
\hline 5 & $1(0,56)$ & $1(1,39)$ & $1(5,88)$ & 0 & \\
\hline 6 & $1(0,56)$ & 0 & 0 & 0 & \\
\hline
\end{tabular}


Para cinco itens do PHQ-9 - sentir-se "para baixo"/ deprimido(a)/sem perspectiva $(p<0,01)$; dificuldade para pegar no sono/permanecer dormindo/dormir mais do que de costume $(p<0,01)$; cansaço/pouca energia $(p<0,01)$; falta de apetite/comendo demais $(p<0,01)$; e sentir-se mal consigo mesmo/achar que é um fracasso/ que decepcionou a família ou a si mesmo $(p=0,03)$ - verificou-se também associação com o gênero feminino. O tipo de instituição foi significativo para o item relativo à ideação suicida ("pensar em se ferir de alguma maneira ou que seria melhor") ( $p=0,03)$, com maior frequência de respostas na instituição privada. Em relação à área do curso dois itens demonstraram esta associação - dificuldade para pegar no sono ou permanecer dormindo, ou dormir mais do que de costume $(p=0,03)$; e falta de apetite/comendo demais $(p<0,01)$.

\section{Discussão}

O objetivo principal deste estudo foi verificar a prevalência de sintomas ansiosos e depressivos em uma amostra de universitários do interior de São Paulo. Nas faixas moderadas e graves, os sintomas ansiosos foram encontrados em $10,75 \%$ dos estudantes e os depressivos em 5,38\% e 5,02\% de acordo com o BDI e o PHQ-9, respectivamente. Em relação à associação das variáveis investigadas com os sintomas apresentados, verificase a predominância do gênero feminino em maiores escores e frequência de respostas em determinados itens para os três instrumentos utilizados. Em relação às demais variáveis, apenas o tipo de instituição demonstrou associação com os escores totais de ansiedade, com alguns itens relacionados ao tipo de instituição e área do curso.

Os resultados apresentados demonstram índices de ansiedade e depressão, nas faixas moderadas e graves, menores do que os encontrados comumente na literatura, aproximando-se dos resultados obtidos de Brandtner e Bardagi (2009) e Simić-Vukomanović et al. (2016). As comparações entre as taxas de sintomas ansiosos e depressivos devem ser feitas com cautela, uma vez que diversos instrumentos e metodologias são empregadas neste tipo de estudo, como diferentes procedimentos de coleta de dados ou cortes utilizados para as faixas de gravidade. Associações entre essas sintomatologias e a variável sexo são vastamente estudadas na literatura, por outro lado, com relação à metodologia e a associação com as demais variáveis nesse estudo, são mais escassos e divergentes.

Os instrumentos aplicados não diagnosticam sozinhos transtornos depressivos ou ansiosos, mas escores acima da gravidade moderada podem indicar a instalação dos mesmos no caso do BAI e BDI, e a presença de sintomas de depressão maior obtido através do PHQ-9 também é outro indicativo de como se encontra a saúde mental dessa população. Os sintomas de ansiedade foram mais prevalentes na amostra estudada do que os de depressão, considerando as faixas sintomáticas relevantes. Os resultados sugerem dificuldades psicológicas importantes na amostra estudada, se consideramos também a ideação suicida presente na amostra, mais de duas vezes maior do que as encontradas por Eisenberg et al. (2007).

A associação significativa dos sintomas com o gênero feminino corrobora a literatura prévia (Baader et al., 2014; Bayram \& Bilgel, 2008; Brandtner \& Bardagi, 2009; Eisenberg et al., 2007; Ibrahim et al., 2013; Mahmoud, Staten \& Lennie, 2012; SimićVukomanović et al., 2016; Steptoe et al., 2007; Weitzman, 2004; Whisman \& Richardson, 2015). Nove sintomas de ansiedade e oito de depressão possuem associação com essa variável. Cinco destes sintomas depressivos (autocrítica, chorar, irritabilidade, mudança na autoimagem, falta de energia e diminuição do interesse sexual) também foram significativamente mais pontuados por mulheres entre 18 e 24 anos no estudo de Molina et al. (2014).

No início da vida adulta, a prevalência de depressão é duas vezes maior nas mulheres, taxas superiores às masculinas desde a adolescência, quando costuma ocorrer o primeiro episódio depressivo. Se considerarmos que um episódio passado é o melhor preditor para um transtorno futuro, esta pode ser uma possível razão para a diferença encontrada nos estudos com populações adultas, mas ainda assim não explica suas causas (Keita, 2007). Os dados de literatura não discutem com clareza sobre fatores causais ou relacionados à vulnerabilidade feminina para ansiedade e depressão, especialmente no que diz respeito ao contexto universitário. No entanto, algumas questões podem ser consideradas. Keita (2007) indica que as mulheres tendem a sofrer mais frequentemente diferentes tipos de eventos negativos, como abuso físico e sexual, pobreza e discriminação de gênero. Molina et al. (2014) também sugerem que as mulheres sejam mais conscientes e incentivadas culturalmente a serem mais observadoras e autocríticas. No sentido inverso, os homens experimentariam de maneira diferente alguns sintomas, considerando, ainda, que a procura masculina por atendimento é mais difícil, fazendo com que a menor prevalência de sintomas encontrada entre indivíduos do gênero masculino não signifique necessariamente menor sofrimento psicológico.

Em relação ao ano da graduação não foram observadas associações, diferindo de outros resultados 
(Brandtner \& Bardagi, 2009; Draken et al., 2012; Mahmoud et al., 2012; Simić-Vukomanović et al., 2016). No entanto, apesar do considerável número de participantes deste estudo, a pouca representatividade de algumas áreas e dos anos finais deve ser considerada quando os resultados são avaliados, sendo esta uma limitação deste trabalho. Nesse sentido, informações referentes à moradia, condição socioeconômica, trabalho, estado civil e histórico de doenças mentais ou de uso medicamentos psiquiátricos poderiam ter sido coletadas a partir da utilização de um questionário sociodemográfico mais completo, enriquecendo as possíveis análises.

Ao contrário de Bayram e Bilgel (2008), Brandtner e Bardagi (2009) e Simić-Vukomanović et al. (2016), não foram encontradas associações entre a área do curso e a sintomatologia ansiosa e depressiva. O tipo de instituição, no entanto, demonstrou associação com os escores totais para sintomas de ansiedade. Não foram encontrados estudos comparativos em relação a esta variável, provavelmente devido às diferenças culturais quanto ao perfil de instituições públicas e privadas conforme variam os países. Além disso, pesquisas em diferentes instituições e locais podem ser mais complexas e onerosas, desincentivando trabalhos do tipo.

\section{Considerações finais}

Os dados demonstram que a população universitária estudada experimenta sintomas ansiosos e depressivos clinicamente significativos, podendo apresentar algum tipo de transtorno ansioso ou depressivo. Além disto, escores totais e sintomas específicos foram associados com a variável gênero, demonstrando a especificidade destas condições. A vulnerabilidade em relação ao gênero feminino é evidente neste estudo e na literatura da área, demonstrando claramente que os programas devem atuar de maneira diferenciada com esta população, focando também em estratégias de prevenção acerca das possíveis causas deste fenômeno.

As instituições de ensino superior devem considerar os dados obtidos pelos estudos da área, procurando aprimorar programas de acolhimento e atendimento - não apenas psicológico - principalmente para os alunos de início do curso. Algumas estratégias podem ser exploradas, como ações preventivas; palestras em aula ou campanhas sobre temáticas relacionadas às demandas do ensino superior e dos suportes oferecidos; engajamento e orientação dos professores e representantes de sala sobre as políticas adotadas na instituição e programas disponíveis; divulgação em aula acerca dos centros de acolhimento psicológico e da importância da procura; e o próprio aperfeiçoamento dos centros disponíveis, focando na identificação dos indivíduos de risco e nos encaminhamentos necessários.

É importante destacar que, apesar deste estudo não possuir como objetivo a investigação de quadros clínicos de ansiedade e depressão, os instrumentos utilizados fora de avaliações clínicas não são suficientes para o estabelecimento de diagnósticos. Optou-se pelo estudo de levantamento, e não por avaliações clínicas, pois o quadro geral de distribuição desses sintomas na população investigada e os indicativos de sintomatologias clínicas que reforcem a necessidade de atenção e mudanças na saúde mental constituem-se como o escopo deste trabalho. Estudos longitudinais também são de suma importância para a avaliação dos alunos ao longo da graduação, verificando quais os períodos mais críticos e/ou quais as especificidades apresentadas em cada etapa da graduação. É importante destacar que os estudos da área seguem metodologias e instrumentos diversos, o que dificulta as comparações a serem feitas, especialmente com relação às variáveis estudadas, característica que poderia ser aprimorada em estudos futuros

Além disso, destaca-se a importância de pesquisas que busquem o aprimoramento dos serviços de suporte oferecidos nas instituições universitárias, considerando sua importância no acolhimento e adaptação desses jovens no contexto universitário, especialmente no início do curso (Viana \& Andrade, 2012), quando rupturas importantes, como a saída da escola, a mudança dos pares e a necessidade de novas posturas frente aos novos eventos sociais e acadêmicos podem agravar sintomas prévios (Brandtner \& Bardagi, 2009). Essas ações preparatórias podem e deveriam se iniciar na idade escolar Bardagi e Hutz (2012), considerando os possíveis impactos da morbidade psicológica (e não apenas de transtornos instalados) na adaptação desta complexa fase.

Agradecimentos pelo auxílio financeiro a Coordenação de Aperfeiçoamento de Pessoal de Nível Superior (CAPES), ao Conselho Nacional de Desenvolvimento Científico e Tecnológico (CNPq) e a Fundação de Amparo à Pesquisa do Estado de São Paulo (FAPESP). 


\section{Referências}

Alvi, T., Assad, F., Ramzan, M., \& Khan, F. A. (2010). Depression, anxiety and their associated factors among medical students. Journal of the College of Physicians and Surgeons Pakistan, 20(2), 122-126. doi:02.2010/JCPSP.122126

American College Health Association (2014). Hanover (MD): American College Health Association - National College Health Assessment II: Reference group executive summary spring 2014. Retrieved from http://www.acha-ncha.org/ docs/ACHA-NCHA-II ReferenceGroup ExecutiveSummary Spring2014.pdf. (DOI INEXISTENTE)

American Psychiatric Association (2013). Manual diagnóstico e estatístico dos transtornos mentais, (5. ed). (DSM-5). Porto Alegre, RS: Artmed. (DOI INEXISTENTE)

Arnett, J. J. (2000). Emerging adulthood: a theory of development from the late teens through the twenties. American Psychologist, 55(5), 469-480. doi:10.1037//0003-066X.55.5.469

Arnett, J. J. (2005). The developmental context of substance use in emerging adulthood. Journal of Drug Issues, 35(2), 235-254. doi:10.1177/002204260503500202

Baader, T. M., Rojas, C. C., Molina, J. L. F., Gotelli, M. V., Alamo, C. P., ... Dittus, P. B. (2014). Diagnóstico de la prevalencia de trastornos de la salud mental en estudiantes universitarios y los factores de riesgo emocionales asociados. Revista Chilena de Neuro-psiquiatría, 52(3), 167-176. doi:10.4067/S0717-92272014000300004

Bardagi, M. P., \& Hutz, C. S. (2012). Rotina acadêmica e relação com colegas e professores: impacto na evasão universitária. PSICO, 43(2), 174-184. (DOI INEXISTENTE)

Bayram, N. \& Bilgel, N. (2008). The prevalence and socio-demographic correlations of depression, anxiety and stress among a group of university students. Social Psychiatry and Psychiatric Epidemiology, 43, 667-672. doi: 10.1007/ s00127-008-0345-x

Brandtner, M. \& Bardagi, M. (2009). Sintomatologia de depressão e ansiedade em estudantes de uma universidade privada do Rio Grande do Sul. Revista Interinstitucional de Psicologia, 2(2), 81-91. (DOI INEXISTENTE).

Cunha, J. A. (2001). Manual da versão em português das Escalas Beck. São Paulo, SP: Casa do Psicólogo.

Draken, A. A. S., Jr, M. H., Rush, B. R., \& Reisbig, A. M. J. (2012). Predictors of anxiety and depression in veterinary medicine students: a four-year cohort examination. Journal of Veterinary Medical Education, 39(4), 322-330. doi:10.3138/jvme.0112-006R

Eisenberg, D., Gollust, S. E., Golbernstein, E., \& Hefner, J. L. (2007). Prevalence and correlates of depression, anxiety, and suicidality among university students. American Journal of Orthopsychiatry, 77(4), 534-542. doi:10.1037/00029432.77.4.534

Hunt, J. \& Eisenberg, D. (2010). Mental health problems and help-seeking behavior among college students. Journal of Adolescent Health, 46, 3-10. doi:10.1016/j.jadohealth.2009.08.008

Fórum Nacional de Pró-Reitores de Assuntos Comunitários e Estudantis [FONAPRECE]. (2011). Perfil socioeconômico e cultural dos estudantes de graduação das instituições federais de ensino superior. Brasília, DF: ANDIFES Recuperado de http://www.andifes.org.br/wp-content/files_flutter/1377182836Relatorio_do_perfi_dos_estudantes_ nas_universidades_federais.pdf.

Huot, K. L., Lutfiyya, M. N., Akers, M. F., Amaro, M. L., Swanoski, M. T., \& Schweiss, S. K. (2013). A populationbased cross-sectional study of health service deficits among U.S. adults with depressive symptoms. Health Services Research, 13, 1-15. doi:10.1186/1472-6963-13-160

Ibrahim, A. K., Kelly, S. J., Adams, C. E., \& Glazebrook, C. (2013) A systematic review of studies of depression prevalence in university students. Journal of Psychiatric Research, 47, 391-400. doi:10.1016/j.jpsychires.2012.11.015

Institute of Medicine and National Research Council (2014). Investing in the health and well-being of young adults. Washington, DC: The National Academies Press. Retrieved from http://www.nap.edu/catalog.php?record_id=18869. (DOI INEXISTENTE)

Instituto Nacional de Estudos e Pesquisas Educacionais Anísio Teixeira (2015). Censo da Educação Superior 2013: resumo técnico. Brasília, DF: INEP. Recuperado de http://download.inep.gov.br/download/superior/censo/2013/ resumo_tecnico_censo_educacao_superior_2013.pdf. (DOI INEXISTENTE)

Keita, P. G. (2007). Psychosocial and cultural contributions to depression in women: considerations for women midlife and beyond. Journal of Managed Care Pharmacy, 13(9), 12-15. doi:10.18553/jmcp.2007.13.9-a.12

Kroenke, K., Spitzer, R. L., \& Williams, J. B. W. (2001). The PHQ-9: Validity of a brief depression severity measure. Journal of General Internal Medicine, 16, 606-613. doi:10.1046/j.1525-1497.2001.016009606.x

Lovell, G. P., Nash, K., Sharman, R., \& Lane, B. R. (2014). A cross-sectional investigation of depressive, anxiety, and stress symptoms and health-behavior participation in Australian university students. Nursing and Health Sciences, 17(1), 134-142. doi:10.1111/nhs. 12147

Mahmoud, J. S. R., Staten, R. T., Hall, L. A., \& Lennie, T. A. (2012). The relationship among young adult college students' depression, anxiety, stress, demographics, life satisfaction, and coping styles. Issues in Mental Health Nursing, 33, 149-156. doi:10.3109/01612840.2011.632708

Molina, M. A. L., Jansen, K., Drews, C., Pinheiro, R., Silva, R., \& Souza, L. (2014). Major depressive disorder symptoms in male and female young adults. Psychology, Health \& Medicine, 19(2), 136-145. doi:10.1080/13548506.2013.793369

Munhoz, T. N., Santos, I. S., \& Matijasevich, A. (2013). Major depressive episode among brazilian adults: A crosssectional population-based study. Journal of Affective Disorders, 150(2), 401-407. doi:10.1016/j.jad.2013.04.031 
Neinstein, L. S. (2013). The new adolescents: An analysis of health conditions, behaviors, risks, and access to services among emerging young adults. Recuperado de https://eshc.usc.edu/thenewadolescents/doc/TheNewAdolescents Final_Locked.pdf (DOI INEXISTENTE)

Sadock, M. J. \& Sadock, V. A. (2007). Compêndio de Psiquiatria: Ciência do comportamento e psiquiatria clínica (9. ed). Porto Alegre, RS: Artmed.

Simić-Vukomanović, I., Mihajlović, G., Kocić, S., Djonović, N., Banković, D., Vukomanović, V., \& Djukić-Dejanović, S. (2016). The prevalence and socioeconomic correlates of depressive and anxiety symptoms in a group of 1,940 Serbian university students. Vojnosanitetski Pregled, 73(2), 169-177. doi:10.2298/VSP141106143S

Somers, J. M., Goldner, E. M., Waraich, P., \& Hsu, L. (2006). Prevalence and incidence studies of anxiety disorders: A systematic review of the literature. The Canadian Journal of Psychiatry, 51(2), 100-113. doi:10.1177/070674370605100206

Spitzer, R. L., Kroenke, K., \& Williams, J. B. W. (1999). Validation and utility of a self-report version of PRIME-MD: the PHQ Primary Care Study. The Journal of the American Medical Association, 282(18), 1737-1744. doi:10.1001/ jama.282.18.1737

Spitzer, R. L., Williams, J. B. W., Kroenke, K., Hornyak, R., \& McMurray, J. (2000). Validity and utility of the Patient Health Questionnaire in assessment of 3000 obstetrics-gynecologic patients. American Journal of Obstetrics \& Gynecology, 183(3), 759-769. doi:10.1067/mob.2000.106580

Steptoe, A., Tsuda, A., Tanaka, Y., \& Wardle, J. (2007). Depressive symptoms, socio-economic background, sense of control, and cultural factors in university students from 23 countries. International Journal of Behavioral Medicine, 14(2), 97-107. doi:10.1007/BF03004175

Viana, M. C. \& Andrade, L. H. (2012). Lifetime prevalence, age and gender distribution and age-of-onset of psychiatric disorders in the São Paulo metropolitan area, Brazil: Results from the São Paulo Megacity Mental Health Survey. Revista Brasileira de Psiquiatria, 34, 249-260. doi:10.1016/j.rbp.2012.03.001

Weitzman, E. R. (2004). Poor mental health, depression, and associations with alcohol consumption, harm, and abuse in a national sample of young adults in college. Journal of Nervous \& Mental Disease, 192(4), 269-277. doi:10.1097/01. nmd.0000120885.17362.94

Whisman, M. A. \& Richardson, E. D. (2015). Normative data on the Beck Depression Inventory - Second Edition (BDI-II) in college students. Journal Of Clinical Psychology, 71(9), 898-907. doi:10.1002/jclp.22188

Dados dos autores:

Juliana Maltoni - Mestre, Faculdade de Filosofia, Ciências e Letras de Ribeirão Preto da Universidade de São Paulo.

Priscila de Camargo Palma - Doutora, Faculdade de Filosofia, Ciências e Letras de Ribeirão Preto da Universidade de São Paulo.

Carmen Beatriz Neufeld - Pós-Doutorado, Faculdade de Filosofia, Ciências e Letras de Ribeirão Preto da Universidade de São Paulo.

\section{Endereço para correspondência:}

Juliana Maltoni

Faculdade de Filosofia, Ciências e Letras de Ribeirão Preto

Departamento de Psicologia

Av. Bandeirantes, 3900, sala 29 - Vila Monte Alegre

14040-900, Ribeirão Preto, SP, Brasil

E-mail: julianamaltoni@gmail.com

Recebido em: 20/11/2017.

Aceito em: 10/09/2018.

Publicado em: 09/05/2019. 